\title{
Design of a non-invasive sensing system for diagnosing gastric disorders
}

\author{
Rosario Morello ${ }^{1}$, Laura Fabbiano ${ }^{2}$, Paolo Oresta ${ }^{2}$, Claudio De Capua ${ }^{1}$ \\ ${ }^{1}$ DIIES, University Mediterranea of Reggio Calabria, Italy \\ ${ }^{2}$ DMMM, Politecnico di Bari University, Italy
}

\begin{abstract}
Gastric disorders are widely spread among the population of any age. At the moment, the diagnosis is made by using invasive systems that cause several side effects. The present manuscript proposes an innovative non-invasive sensing system for diagnosing gastric dysfunctions. The Electro-GastroGraphy (EGG) technique is used to record myoelectrical signals of stomach activities. Although EGG technique is well known for a long time, several issues concerning the signal processing and the definition of suitable diagnostic criteria are still unresolved. So, EGG is to this day a trial practice. The authors want to overcome the current limitations of the technique and improve its relevance. To this purpose, a smart EGG sensing system has been designed to non-invasively diagnose gastric disorders. In detail, the system records the gastric slow waves by means of skin surface electrodes placed in the epigastric area. Cutaneous myoelectrical signals are so acquired from the body surface in proximity of stomach. Electro-gastrographic record is then processed. According to the diagnostic model designed from the authors, the system estimates specific diagnostic parameters in time and frequency domains. It uses Discrete Wavelet Transform to obtain power spectral density diagrams. The frequency and power of the EGG waveform and the dominant frequency components are so analyzed. The defined diagnostic parameters are put in comparison with the reference values of a normal EGG in order to estimate the presence of gastric pathologies by the analysis of arrhythmias (tachygastria, bradygastria and irregular rhythm). The paper aims to describe the design of the system and of the arrhythmias detection algorithm. Prototype development and experimental data will be presented in future works. Preliminary results show an interesting relevance of the suggested technique so that it can be considered as a promising non-invasive tool for diagnosing gastrointestinal motility disorders.
\end{abstract}

Section: RESEARCH PAPER

Keywords: Gastric disorders; gastric slow wave; EGG; myoelectrical measurements

Citation: Rosario Morello, Laura Fabbiano, Paolo Oresta, Claudio De Capua, Design of Design of a non-invasive sensing system for diagnosing gastric disorders, Acta IMEKO, vol. 10, no. 4, article 14, December 2021, identifier: IMEKO-ACTA-10 (2021)-04-14

Section Editor: Francesco Lamonaca, University of Calabria, Italy

Received October 2, 2021; In final form October 24, 2021; Published December 2021

Copyright: This is an open-access article distributed under the terms of the Creative Commons Attribution 3.0 License, which permits unrestricted use, distribution, and reproduction in any medium, provided the original author and source are credited.

Corresponding author: Rosario Morello, e-mail: rosario.morello@unirc.it

\section{INTRODUCTION}

The rapid advancement of information technologies now allows the physicians to be able to smartly follow and assist the patient in real time even remotely through simple dedicated applications [1]-[3]. In the same perspective, the case of gastrointestinal pathologies has been addressed here.

Dyspepsia, stomach ulcer, gastritis, esophageal reflux are some examples of gastrointestinal motility disorders. Such pathologies are widely spread among the population and their symptoms can become strongly debilitating. Gastric disorders include several dysfunctions of the stomach digestive activity. Gastroesophageal scintigraphy and endoscopy (gastroscopy) are at the moment two invasive techniques extensively used in the practice to diagnose gastric disorders. During the digestive function, the stomach muscles contract rhythmically to allow digestive activity to be performed. This activity is regulated from myoelectrical waves. Such waves in presence of additional stimuli induce muscles to contract, [4]-[8]. Electromyographic measurements of such gastric slow waves can provide important information on the stomach activity, [9]. Electrogastrography (EGG) is a technique known from several years based on recording stomach muscle contractions by means of skin electrodes, [10], [11]. Such technique suffers from inappropriate data processing algorithms and interpretation errors, so showing poor reliability in the use of it as a diagnostic method of gastrointestinal motility disorders. Nevertheless, recent medical trials have highlighted a clear correlation between abnormal gastric electrical activity and the onset of specific dysfunctions, 
[12], so that the gastroenterologists have recently reconsidered it as a potential non-invasive screening technique. In addition, the American Gastroenterological Association (AGA) states the clinical relevance of EGG in demonstrating gastric myoelectric abnormalities in patients with unexplained nausea and vomiting or functional dyspepsia, [13], [14]. It represents a promising and interesting alternative method for gastric screening since it has no side effects and is painless, [15]. Nevertheless, further advances on processing algorithms and on the project of more accurate measurement systems are required in order to improve the reliability and evidence of the method, [16]. At present, there are no standardized diagnostic criteria and the state of art highlights poor care about this issue. Therefore, several aspects must be still investigated, and additional studies are needed to assess the use of EGG as an alternative test to the actually used invasive techniques, [17]-[19]. As it has been assessed above, an EGG system records the stomach myoelectrical activity by means of cutaneous leads placed over the gastric area. In this way, it is possible to estimate patient's gastrointestinal conditions by analysing the slow waves in time and frequency domains. In presence of gastric disorders, myoelectrical abnormalities can be revealed and characterized in the EGG records, due to a decreased activity of the stomach muscles and nerves. In healthy individuals, standard EGG record is characterized by regular electrical rhythm. In detail, it consists of periodic waveforms with a predominant frequency of 3 cycles per minute (cpm) at rest. During the digestive activity, frequency and intensity of gastric waves increase. In individuals suffering from gastrointestinal motility disorders, electro-gastrographic measurements have instead an irregular rhythm. In addition, post meal, sometimes, no increase of frequency and intensity of the waveform is observed. These and further features must be analysed in order to define suitable diagnostic criteria for characterizing the occurrence of gastric motility disorders. Another interesting application of the electro-gastrographic technique concerns the study of patients affected from vomiting, unexplained nausea, improper digestion of food and gastroparesis. Medical trials have been carried out to get important information on the mechanism that regulates the activity of stomach muscles and nerves in presence of those disorders, [19]-[21]. So, for example, it can be an helpful technique in understanding the origin of the unexplained contractions which cause vomiting in patients affected by anorexia, [22], [23]. That would allow gastroenterologists to schedule new therapies so to reduce the vomiting stimulus.

Therefore, more and more physicians show renewed interest in this technique. Nevertheless, careful studies have still to highlight its potentialities. In this sight, the authors have focused their research activity on such aspects in order to overcome limitations and lack in the interpretation of the EGG waveforms. So, the authors have proposed in [24] an innovative diagnostic model for characterizing gastric myoelectrical abnormalities due to disorders, and gained long-standing experience in recording of myoelectrical signals, [25]-[27].

In the present manuscript, the authors describe the developments of the model previously proposed. In detail, a smart and automated EGG sensing system has been designed and its project is described in the following. By the embedded diagnostic criteria, the system is able to recognize an abnormal gastric activity. The used methodical approach starts with the study of the electro-gastrographic technique. Standard EGG records of healthy persons have been analysed in order to define suitable diagnostic reference parameters. Such parameters contribute to define and recognize the onset of gastric disorders. Then, diagnostic criteria have been defined to optimize the analysis of the EGG waves in patients affected from gastric disorders. The diagnosis is based on a multifactorial analysis of the defined diagnostic parameters, which are compared with the respective reference values of a standard EGG signal. The EGG sensing system has been projected and developed according to the Measurement System Design Model described in [28]. The system acquires and processes gastric myoelectrical waves in compliance with the diagnostic model presented in [24]. Then the system decides among five alternative diagnoses.

In the next Section an overview of the electro-gastrographic technique is reported, and main gastric disorders and some applications of EGG in medical practice are described. The third and fourth Sections respectively analyses the phenomenon and describes the project of the smart EGG sensing system and the embedded diagnostic algorithm. Next, some results are presented, and Conclusions follow.

\section{ELECTROGASTROGRAPHY}

The electro-gastrographic technique is known in medical field for a long time. It has common features with the electrocardiogram, as both techniques are based on myoelectrical signal measurements. The EGG is a non-invasive technique based on recording the gastric myoelectrical activity. Now, it cannot be considered in effect as a diagnostic tool because of lack of its standardization. Inaccurate instrumentation, interpretation errors and lack of approved diagnostic criteria are some reasons. So, the authors have carefully examined the state of art of the technique in order to understand the current use of it. The method has been used in medical practice to study patients affected from unexplained persistent or episodic symptoms related to gastric motility disorders. Further studies have been carried out by analysing gastric waveforms of patients with unexplained nausea and vomiting. They have shown interesting and promising results. The same analysis cannot be performed by means of invasive diagnostic tools, such as endoscopy, because of the artefacts introduced during the examination. In fact, endoscopy can be cause of further vomiting stimuli which overlap to patient's nausea. Other clinical trials highlight that functional dyspepsia and gastroparesis can be characterized by analysing gastric myoelectrical activity. In such cases, arrhythmias of the EGG waveform can be clearly observed. Further studies have singled out the occurrence of abnormalities in the EGG waves of patients with other specific gastric disorders such as stomach ulcer, gastritis, oesophageal reflux, early satiety, anorexia. Experimental results have shown an interesting correlation between gastric myoelectrical impulses and stomach diseases. For this reason, this technique can be considered as a promising and practical screening tool for the evaluation of several gastrointestinal motility disorders. Nevertheless, before considering EGG as a reliable diagnostic test, several aspects need still to be explained and highlighted. The authors have so focused their attention on these issues. The final aim is to propose the project of non-invasive sensing system with embedded diagnostic criteria. In order to use a methodical approach to the matter, we must understand the mechanism which regulates the gastric myoelectrical impulses and the stomach muscles contraction. Therefore, the behaviour of the stomach during gastric function (digestion) in healthy persons has been analysed. Once the myoelectrical activity of the stomach has been investigated, it has been possible to characterize the 


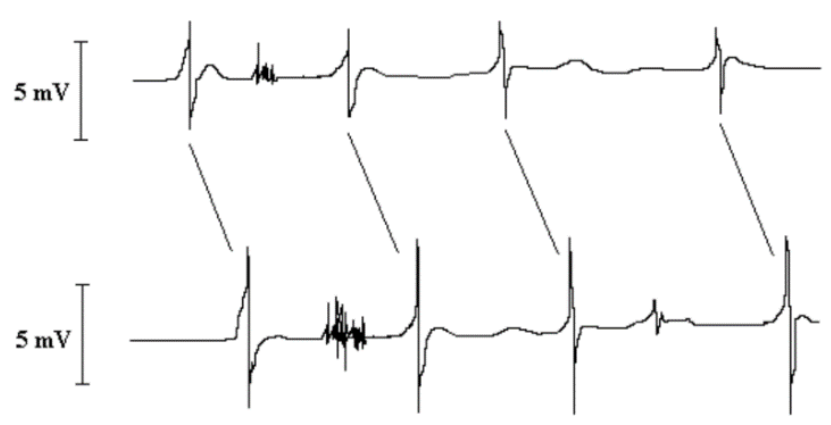

Figure 1. EGG waveform.

standard EGG waveform so to correctly interpret the occurrence of possible abnormalities in the myoelectrical activity, [29]-[31].

In detail, stomach's muscles contraction and nerves movement are regulated by myoelectrical impulses, [30], [31] Such gastric waves control and coordinate the stomach activity. Periodic waves, within specific frequency and amplitude ranges, are usual and allow stomach to digest food. Electrogastrographic signals have amplitude relatively low, about $200-$ $5000 \mu \mathrm{V}$. Consequently, the acquired signal must be amplified before of the processing stage. The frequency range is $0.016-$ $0.25 \mathrm{~Hz}$ equal to $1-15 \mathrm{cpm}$, see Figure 1 for reference.

At rest, slow waves depolarize the gastric smooth muscles without causing contraction. Amplitude of EGG waves increases, with the ingestion of food and when digestion starts, due to the increased activity of the stomach's muscles. During digestion, indeed, the contraction of the muscles is caused by additional depolarization. So gastric slow waves control the fundamental frequency and the direction of contraction. This behaviour describes the regular mechanism of the gastric function.

Differently, in presence of gastrointestinal disorders, arrhythmias can be observed in the EGG waveform due to an incorrect stomach's activity. Such abnormal myoelectrical activity is cause of changes of the fundamental frequency component and of its intensity. For instance, when a reduced contractile function of the stomach is observed, the EGG waveform is characterized by lower frequency values of the fundamental component known as bradygastria. It is due to a reduced number of contractions. Conversely, higher frequency values of the fundamental component, or tachygastria, cause stomach atonicity. Generally, the pathogenesis of the gastric myoelectrical arrhythmias is due to the delayed stomach emptying which occurs when the individual is affected from motility gastrointestinal disorders. Consequently, it is cause of a reduced stomach activity

\section{STANDARD EGG WAVEFORM}

Clinicians suggest collecting EGG recordings after overnight fasting and during food digestion, in order to analyse the gastric function at rest and during digestive activity. The patient must be in a comfortable position to prevent movement artefacts and should remain motionless during the whole EGG acquisition. A preliminary recording is performed with empty stomach, 15 to 60 minutes. Subsequently the patient must consume a caloric meal (300 kcal), and a further EGG 30 to 120 minutes recording is acquired. Normally physicians suggest a fasting recording of 30 minutes and a postprandial recording of 60 minutes. In this way, it is possible to evaluate the gastric response during meal digestion. A set of EGG signals, recorded during fasting and postprandial stages, has been analysed in time and frequency domains by using Discrete Wavelet Transform. Frequency components and their amplitude (power) have been considered. By means of power/frequency spectral analysis, the postprandial and fasting records have been compared. Commonly it is assumed that a normal EGG waveform is characterized by an averaged dominant frequency of about $3 \mathrm{cpm}$. During digestion, both frequency and associated amplitude increase. Rhythm abnormalities include bradygastria (lower dominant frequency), tachygastria (higher dominant frequency), and irregular rhythm (dysrhythmia). Nevertheless, such averaged values are not reliable because they can significantly change from individual to individual (physical constitution, age, general healthy status, etc.). Consequently, a careful study of the literature and further analyses of EGG signals of healthy individuals have allowed us to characterize a reference model. Two quantities must be considered: frequency and amplitude. In a standard EGG waveform, the fasting dominant frequency of gastric waves has to belong to the interval $2-4 \mathrm{cpm}$. In the postprandial recording, dominant frequency must belong to the normal frequency range 2-4 cpm for at least $75 \%$ of time. This percentage time depends on the type of meal consumed. If the dominant frequency belongs to the previous interval only for $25 \%$ of the EGG recording time, then it can be considered index of dysrhythmia. This occurrence happens because of an altered gastric emptying. Lower frequency values or equal to $2 \mathrm{cpm}$ are index of bradygastria. Higher frequency values or equal to $4 \mathrm{cpm}$ define tachygastria. In a regular recording segment, different zones with bradygastria and tachygastria may be characterized. Dysrhythmia can be characterized if the relative abnormal frequency waveform takes at least 5 minutes. The recognition of these patterns is simple, the only parameter to be considered is the dominant frequency value. Further relevant parameters in the time domain can be used to characterize an irregular gastric activity. For example, an abnormal EGG record can be characterized by the presence of bradygastria or/and tachygastria regions over $30 \%$ of the recording time. As an alternative, the EGG waveform can be considered irregular if the percentage of power distribution in the bradygastria or tachygastria regions is greater than $20 \%$. With reference to the signal intensity, we can estimate the absolute amplitude or power of EGG waveform by means of the weighted summation of the gastric waves. Differently, the percentage power distribution is obtained by summing waveform power for each frequency band and dividing by the total signal power of the recording, the result is multiplied by 100 . Typically, a power ratio between postprandial and fasting signals lower or equal to 1 may suggest a decreased gastric response to the meal. On the contrary, it is expected an increase in the myoelectrical activity of the stomach during digestion. Finally, nausea and early satiety, are typically cause of gastric dysrhythmia, but this occurrence does not include necessarily an altered gastric emptying rate.

\section{THE SMART EGG SENSING SYSTEM}

In this Section, the project of the EGG sensing system is described. The system has been projected according to the Measurement System Design Model in [28]. Through an algorithm, the smart system is able to extract information from the EGG signal according to the criteria reported in the Table 2. It is a smart and patient-adaptive system which can detect gastrointestinal motility disorders. The system project has a microcontroller architecture in order to manage the data flow 


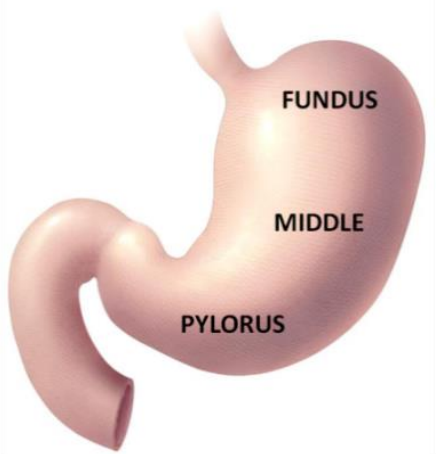

Figure 2. Sections of the stomach.

and the data processing. Three cutaneous $\mathrm{Ag} / \mathrm{AgCl}$ electrodes are used to acquire and record the gastric myoelectrical signals, [32]. Electrodes must be placed on the anterior abdominal wall over the stomach (epigastrium antrum). It is preferable that specialized medical staff perform the electrodes displacement. However, for further information, a brief description of the procedure is provided here. Being the stomach located in proximity of the end of the rib section, it is helpful to divide it in three zones in order to suitably place the electrodes: the fundus (upper region), the stomach body or middle, and the pylorus (end of the stomach), see Figure 2.

Two electrodes must be placed under the ribs in proximity of the fundus and mid corpus of the stomach (along the antral axis); the third one, the ground reference, is placed at the end of the stomach (see black circles in Figure 3). This configuration allows signal-to-noise ratio to be minimized.

Since the electro-gastrographic signal has a relatively low amplitude, it is amplified before of the processing stage by means of an Analog Device Amplifier AD524. According to the frequency range $(1-15 \mathrm{cpm})$, a band pass filter, with cutoff frequencies equal to 0.010 and $0.3 \mathrm{~Hz}$, has been used to eliminate frequency components which are lower than $1 \mathrm{cpm}$ and higher than 15 $\mathrm{cpm}$. In this way, it is possible to remove the baseline drift and to exclude signals from other sources: possible myoelectrical interferences can be due to the heart, colon and small intestine. Further interferences or artefacts are due to breathing, movements or electrical noise. These artefacts have commonly frequency components which are lower than $1 \mathrm{cpm}$ (motion artefacts) and higher than $9 \mathrm{cpm}$ (respiratory artefacts). Such overlapping signals could cause an erroneous estimation of signal amplitude and dominant frequency. Therefore, the filter has been carefully designed to reject both further myoelectrical contributions due to other organs and artefacts and noise. The signal is sampled with a sampling frequency of $2 \mathrm{~Hz}$, and is subsequently processed by a Discrete Wavelet Transform, [33], [34].

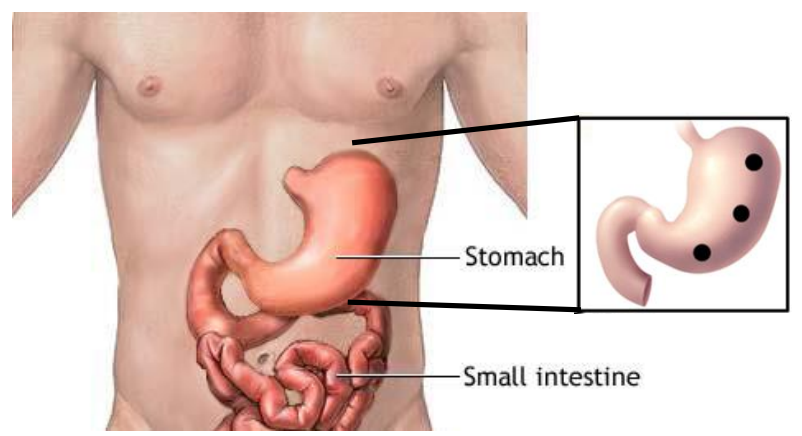

Figure 3. EGG electrodes displacement.
By means of spectral analysis, it is possible to estimate the power and amplitude of the signal frequency components, [35], [36]. The waveform analysis in time and frequency domains allows the system to get information on the power trends as function of frequency and/or time. In this way, it is possible to characterize the arrhythmias of the myoelectrical signal. Specific memory devices have been used to store information concerning the metrological characteristics of the system and patient's clinical history. In detail, information on measurement uncertainty and calibration curve is stored in a first memory device in order to estimate the reliability of measurement results. In addition, a further writable and readable storage device stores private and medical data concerning the case-history of the patient in order to improve the diagnosis reliability. Figure 4 shows the flow diagram of the EGG signal processing.

The amplification and filtering blocks allow the system to perform a preliminary pre-processing of the input signal. The filtering stage performs the rejection of noise and artefact signals overlapped to the EGG waveform. Two amplification stages have been used to amplify the voltage levels. Once the electrodes are properly displaced, the system performs a fasting recording 30 minutes long and a postprandial recording lasting 60 minutes. Subsequently, the acquired signals are processed according to the diagnostic model, [24].

Abnormalities in the EGG record can be characterized by considering the power vs frequency trend. Gastrointestinal motility disorders cause, in fact, arrhythmias that, if characterized by frequency components above the normal range, indicate tachygastria, by frequency components below normal range indicate bradygastria; if several frequency contributions arise, then that indicate dysrbythmia; further, lack of signal power increases during postprandial recording. Consequently, five patterns are considered: i) normal EGG; ii) bradygastria; iii) tachygastria; iv) dysrhythmia; v) lack of postprandial power increase.

The analysis in Section 3 has allowed us to define specific diagnostic parameters which are representative of the gastric myoelectrical signal features, see Table 1.

These parameters provide a complete description of the EGG waveform in terms of spectral and power analysis. Basic requirements are: $T D F, f_{-} T D F$ and $p_{-} T D F$ with a time duration which has to be higher than 5 minutes.

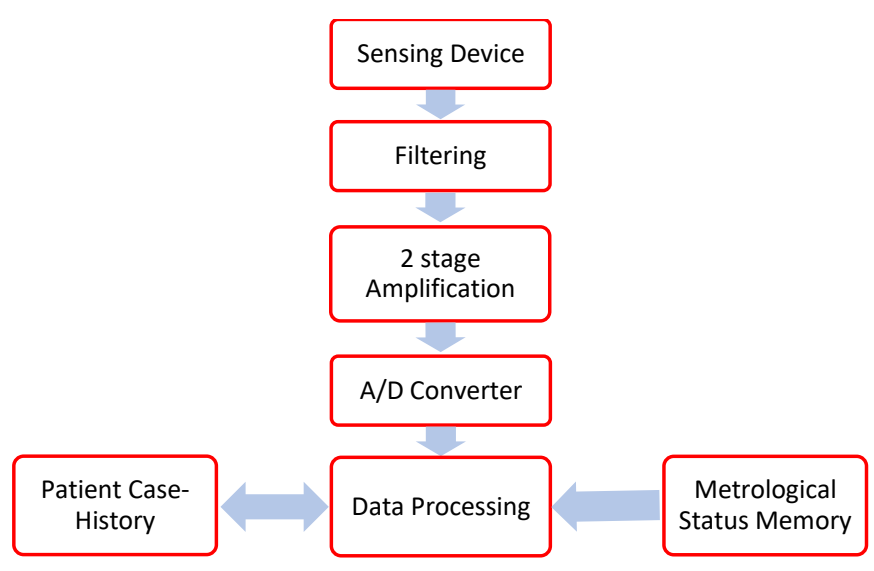

Figure 4. Flow diagram of the EGG signal path. 
Table 1. Defined diagnostic parameters.

\begin{tabular}{ll}
\hline Parameter & Description \\
\hline DF in cpm & Dominant frequency \\
TDF in s & Recording time of dominant frequency \\
PDF in dBm & Power distribution of dominant frequency \\
f_DF in cpm & Fasting dominant frequency \\
__TDF in s & Recording time of fasting dominant frequency \\
f_PDF in dBm & Power distribution of fasting dominant frequency \\
f_T in s & Fasting recording time \\
P_DF in cpm & Postprandial dominant frequency \\
P_TDF in s & Recording time of postprandial dominant frequency \\
P_PDF in dBm & Power distribution of postprandial dominant frequency \\
P_T in s & Postprandial recording time \\
RDF & Ratio of postprandial to fasting power of DF \\
T3F in s & Recording time of [2-4] cpm frequency range \\
P_T3F in s & Recording time of postprandial [2-4] cpm frequency range \\
f_T3F in s & Recording time of fasting [2-4] cpm frequency range \\
P3F in dBm & Power distribution of [2-4] cpm frequency range \\
TTF in s & Total recording time of tachygastria frequency \\
PTF in dBm & Power distribution of tachygastria frequency \\
TBF in s & Total recording time of bradygastria frequency \\
PBF in dBm & Power distribution of bradygastria frequency \\
\%P3F & Percentage of power distribution of [2-4] cpm frequency range \\
\%PTF & Percentage of power distribution of tachygastria frequency \\
\%PBF & Percentage of power distribution of bradygastria frequency \\
\hline
\end{tabular}

Firstly, the embedded algorithm allows the system to estimate the dominant frequency $(D F)$, the time length of dominant frequency recording (TDF), the associated amplitude and power distribution $(P D F)$. In this way it is possible to verify the rhythm of the EGG waveform. In detail, the system estimates the fasting and postprandial dominant frequencies $\left(f_{-} D F, p_{-} D F\right)$ and their recording time $\left(f_{-} T D F, p_{-} T D F, f_{-} T, p_{-} T\right)$, amplitude and power distribution ( $\left.f_{-} P D F, p_{-} P D F\right)$. Subsequently, the ratio of postprandial to fasting power $(R D F)$ is evaluated in order to assess the occurrence of a decreased gastric response to the meal. In order to verify the possible occurrence of dysrbythmias, the recording time and power distribution of EGG frequencies in/above/below the $2-4 \mathrm{cpm}$ intervals are computed (T3F, P_T3F, $\left.f_{-} T 3 F, P 3 F\right)$. The recording time and power distribution of tachygastria and bradygastria ranges (P3F, TTF, PTF, TBF, PBF) are subsequently estimated. Finally, the percentage of power distribution in the three frequency ranges $(\% \mathrm{PF}, \% \mathrm{PTF}, \% \mathrm{PBF})$ can be obtained by estimating the weighted summation of power contributions divided by the total power. The last parameters allow us to characterize the presence of tachygastria and bradygastria patterns.

Once the previous diagnostic parameters have been estimated, the smart EGG sensing system verifies the presence of possible irregular gastric activities. To this aim, each parameter is compared with the homologous reference value of a normal EGG record, described in Section III. Five alternative diagnoses (normal EGG, bradygastria, tachygastria, dysrbythmia and lack of postprandial power increase) are available. Specific conditions about time, frequency and power must be satisfied simultaneously in order to make a specific diagnosis. Table 2 summarizes the embedded diagnosis criteria.

Table 2. Diagnosis criteria.

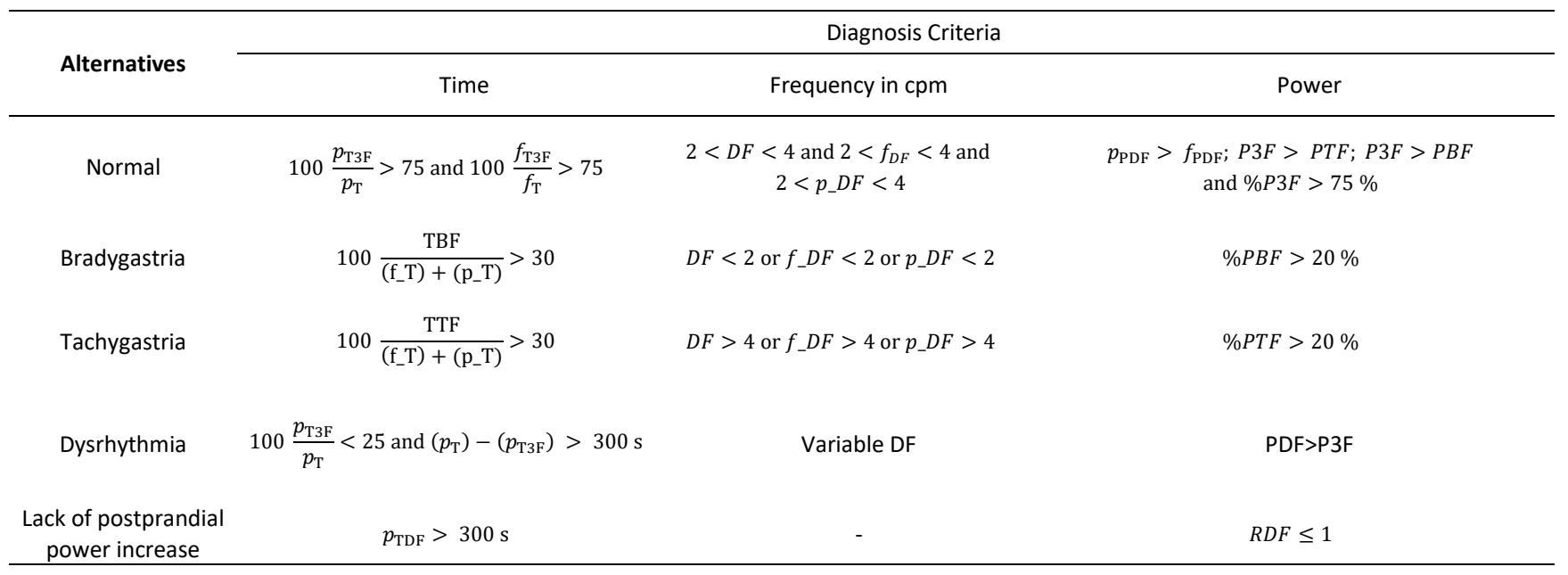




\section{DISCUSSION}

The previous parameters and the diagnosis criteria have been characterized by analysing EGG records of healthy individuals, [24]. Standard EGG signals have been considered to define the normal range of each parameter. The design of the EGG sensing system embeds such diagnosis criteria which have been validated by means of simulations.

Preliminary results have been carried out by using standard EGG records in order to verify the possible occurrence of falsepositive diagnoses. EGG records have been generated by means of an arbitrary waveform generator in laboratory. Twenty cases have been considered. In all cases, the system has properly detected the absence of arrhythmias in compliance with the expected behaviour.

Further tests have been performed to verify the system capability to reject the artefacts. Motion and respiratory artefacts have been reproduced and added to a normal EGG record. The noisy signal has been processed and the artefacts have been properly removed obtaining the initial EGG signal.

Additional simulations have been performed in order to test the sensitivity of the system. EGG records with gastric disorders have been generated in Matlab environment to verify the degree to which the embedded numerical algorithm responds to slight changes of the diagnostic parameters. Each pattern occurrence has been reproduced in order to prove the effectiveness and accuracy of diagnosis results. Then, the signals have been generated by using an arbitrary waveform generator. Therefore, the experimental results do not regard specific patients, but they are the consequence of simulations. The system response has been observed. In detail, EGG waveforms have been generated starting from standard waves. The single diagnostic parameter has been modified with progressive percentage deviations. In this way, it has been possible to characterize the sensitivity of the system. The presence of gastric arrhythmias has been detected in presence of deviations above $7 \%$ of the reference values in Table 2. The EGG system has shown a good capability to detect each pattern. The total sensitivity was above $94 \%$.

\section{CONCLUSIONS}

In this paper the electro-gastrographic technique (EGG) is proposed to record and process myoelectrical signals of the stomach activity. Several studies in literature show an interesting relevance of electro-gastrography to diagnose gastric disorders. Although EGG technique is well known, several issues are still unresolved. So, EGG is, up to day, a trial practice. The authors propose the design of a smart EGG sensing system in order to overcome the current limitations of the technique and improve its relevance and evidence. The system has been projected according to the IEEE 1451 Standard. It is able to acquire the EGG signal by means of skin electrodes and to process it according to the embedded diagnostic model previously defined by the authors. Diagnostic parameters and their reference values have been characterized by analysing EGG records of healthy individuals. By using the diagnostic model, the smart system is able to assess the occurrence of abnormal myoelectrical activity of the stomach due to gastric pathologies. Five alternative diagnoses have been considered: normal EGG, bradygastria, tachygastria, dysrhythmia, lack of postprandial power increase. Preliminary simulations have shown interesting results. In detail, the proposed system has been tested by using normal EGG records and simulated waveforms, so to verify its sensitivity and selectivity. Experimental data have shown promising results.
The proposed sensing system may be considered a noninvasive tool for diagnosing gastrointestinal motility disorders as an alternative to invasive techniques such as gastroscopy.

At the moment, the research activity is expecting for funding in order to develop the system and carry out experimentation on real case-studies. Medical trials have been scheduled and results will be reported in future works.

\section{REFERENCES}

[1] H. M. Shamim, G. Muhammad, A. Alamri, Smart healthcare monitoring: a voice pathology detection paradigm for smart cities. Multimedia Systems 25.5 (2019), 565-575

DOI: $10.1007 / \mathrm{s} 00530-017-0561-\mathrm{x}$

[2] R. Morello, C. De Capua, L. Fabbiano, G. Vacca, Image-based detection of Kayser-Fleischer ring in patient with Wilson Disease, 2013 IEEE International Symposium on Medical Measurements and Applications (MeMeA). DOI: $10.1109 / \mathrm{MeMeA} .2013 .6549715$

[3] G. Muhammad, M. F. Alhamid, X. Long, Computing and processing on the edge: Smart pathology detection for connected healthcare. IEEE Network 33.6 (2019), pp. 44-49. DOI: $10.1109 /$ MNET.001.1900045

[4] J. J. Baker, E. Scheme, K. Englehart, D. T. Hutchinson, B. Greger, Continuous Detection and Decoding of Dexterous Finger Flexions with Implantable MyoElectric Sensors, IEEE Transactions on Neural Systems and Rehabilitation Engineering, Vol. 18, no.4, 2010, pp. 424-432.

DOI: $10.1109 /$ TNSRE.2010.2047590

[5] Byung Woo Lee, Chungkeun Lee, Jinkwon Kim, Jong-Ho Lee, Comparison of Conductive Fabric Electrode with Electromyography to Evaluate Knee Joint Movement, IEEE Sensors Journal, Vol.12, no.2, 2012, pp. 410-411. DOI: $10.1109 /$ JSEN.2011.2161076

[6] G. Imperatori, P. Cunzolo, D. Cvetkov, D. Barrettino, Wireless Surface Electromyography Probes with Four High-Speed Channels, IEEE Sensors Journal, Vol.13, no.8, 2013, pp. 29542961.

DOI: $10.1109 /$ JSEN.2013.2260145

[7] John W. Arkwright, Neil G. Blenman, Ian D. Underhill, Simon A. Maunder, Nick J. Spencer, Marcello Costa, Simon J. Brookes, Michal M. Szczesniak, Phil G. Dinning, Measurement of Muscular Activity Associated with Peristalsis in the Human Gut Using Fiber Bragg Grating Arrays, IEEE Sensors Journal, Vol.12, no.1, 2012, pp. 113-117.

DOI: $10.1109 /$ JSEN.2011.2123883

[8] A. Lay-Ekuakille, P. Vergallo, A. Trabacca, M. De Rinaldis, F. Angelillo, F. Conversano, S. Casciaro, Low-Frequency Detection in ECG Signals and Joint EEG-Ergospirometric Measurements for Precautionary Diagnosis, Measurement, Vol. 46, Issue 1, 2013, pp 97-107.

DOI: $10.1016 /$ j.measurement.2012.05.024

[9] S. Somarajan, N. Muszynski, J. Olson, A. Comstock, A. Russell, L. Walker, S. Acra, L. Bradshaw, The effect of chronic nausea on gastric slow wave spatiotemporal dynamics in children, Neurogastroenterology \& Motility 33.5 (2021): e14035.

DOI: $10.1111 / \mathrm{nmo} .14035$

[10] H. P. Parkman, W. L. Hasler, J. L. Barnett, E. Y. Eaker, Electrogastrography: a document prepared by the gastric section of the American Motility Society Clinical GI Motility Testing Task Force, Neurogastroenterol Motil, Blackwell Publishing Ltd, 2003, pp. 89-102.

[11] B. Pfaffenbach, R. Adamek, K Kuhn, M. Wegener, Electrogastrography in healthy subjects, Digestive Diseases and Sciences Journal, Springer, Vol. 40, Issue 7, 1995, pp. 1445-1450. DOI: $\underline{10.1007 / \mathrm{BF} 02285190}$

[12] C. Varghese, D. A. Carson, S. Bhat, T. C. L. Hayes, A. A. Gharibans, C. N. Andrews, G. O'Grady, Clinical associations of functional dyspepsia with gastric dysrhythmia on 
electrogastrography: A comprehensive systematic review and meta-analysis, Neurogastroenterology \& Motility (2021), e14151. DOI: $10.1111 /$ nmo.14151

[13] Report of American Gastroenterological Association (AGA), American Gastroenterological Association medical position statement: Nausea and vomiting, Gastroenterology, Vol. 120, Issue 1, 2001, pp. 261-263.

[14] A. Ravelli, Gastric Motility and Electrogastrography (EGG). In: H. Till, M. Thomson, J. Foker., G. Holcomb III, K. Khan (eds) Esophageal and Gastric Disorders in Infancy and Childhood. Springer, Berlin, Heidelberg, ISBN : 978-3-642-11201-0.

[15] G. Gopu, R. Neelaveni, K. Porkumaran, Investigation of digestive system disorders using Electrogastrogram, Proc. International IEEE Conference on Computer and Communication Engineering, Kuala Lumpur, 13-15 May 2008, pp 201-205.

DOI: $10.1109 /$ ICCCE. 2008.4580596

[16] F. Y. Chang, C. L. Lu, S. D. Lee, G. L. Yu, An improved electrogastrographic system in measuring myoelectrical parameters, Journal of Gastroenterology and Hepatology, Vol. 13, Issue 10, 1998, pp. 1027-1032.

DOI: $10.1111 / \mathrm{j} .1440-1746.1998 . t b 00565 . x$

[17] J. L. Gonzalez-Guillaumin, D. C. Sadowski, O. Yadid-Pecht, K. V. I. S. Kaler, M. P. Mintchev, Multichannel pressure, bolus transit, and $\mathrm{pH}$ esophageal catheter, IEEE Sensors Journal, Vol.6, no.3, 2006, pp. 796-803.

DOI: $10.1109 /$ JSEN.2006.874437

[18] A. Cysewska-Sobusiak, P. Skrzywanek, A. Sowier, Utilization of Miniprobes in Modern Endoscopic Ultrasonography, IEEE Sensors Journal, Vol.6, no.5, 2006, pp. 1323-1330.

DOI: $\underline{10.1109 / J S E N .2006 .877985}$

[19] J. D. Z. Chen, Non-invasive measurement of gastric myoelectrical activity and its analysis and applications, Proc. 20th International Conference of the IEEE Engineering in Medicine and Biology Society, Vol. 6, Hong Kong, November 1998, pp. 2802-2807.

DOI: $10.1109 /$ IEMBS.1998.746065

[20] R. Yoshida; K. Takahashi; H. Inoue; A. Kobayashi, A study on diagnostic capability of simultaneous measurement of electrogastrography and heart rate variability for gastroesophageal reflux disease, Proc. IEEE SICE Annual Conference (SICE), Akita, Japan, August 2012, pp. 2157-2162.

[21] Zhang-Yong Li, Chao-Shi Ren, Shu Zhao, Hong Sha, Juan Deng, Gastric motility functional study based on electrical bioimpedance measurements and simultaneous electrogastrography, Journal of Zhejiang University - Science B, Springer, Vol. 12, Issue 12, 2011, pp. 983-989.

DOI: $10.1631 /$ jzus.b1000436

[22] D. A. Carson, S. Bhat, T. C. L. Hayes, A. A. Gharibans, C. N. Andrews, G. O'Grady, C. Varghese, Abnormalities on Electrogastrography in Nausea and Vomiting Syndromes: A Systematic Review, Meta-Analysis, and Comparison to Other Gastric Disorders. Dig Dis Sci (2021). DOI: $10.1007 / \mathrm{s} 10620-021-07026-\mathrm{x}$

[23] Panyko, Arpád, Marián Vician, Martin Dubovský, Massive Acute Gastric Dilatation in a Patient with Anorexia Nervosa, Journal of Gastrointestinal Surgery 25.3 (2021), pp. 856-858. DOI: $10.1007 / \mathrm{s} 11605-020-04715-2$

[24] R. Morello, C. De Capua, F. Lamonaca, Diagnosis of Gastric Disorders by Non-invasive Myoelectrical Measurements, Proc. 2013 IEEE International Instrumentation and Measurement Technology Conference (I2MTC 2013), Minneapolis, MN, 6-9 May 2013, pp. 1324-1328. DOI: $10.1109 /$ I2MTC.2013.6555628

[25] C. De Capua, A. Meduri, R. Morello, A Remote Doctor for Homecare and Medical Diagnoses on Cardiac Patients by an
Adaptive ECG Analysis, Proc. IEEE 4th International Workshop on Medical Measurement and Applications (MeMeA 2009), Cetraro, Italy, May 2009, pp.31-36.

DOI: $10.1109 /$ MEMEA.2009.5167949

[26] C. De Capua, A. Meduri, R. Morello, A Smart ECG Measurement System Based on Web Service Oriented Architecture for Telemedicine Applications, IEEE Transactions on Instrumentation and Measurement, Vol. 59, Issue 10, 2010, pp. 2530-2538. DOI: $10.1109 /$ TIM.2010.2057652

[27] C. De Capua, A. Battaglia, A. Meduri, R. Morello, A PatientAdaptive ECG Measurement System for Fault-Tolerant Diagnoses of Heart Abnormalities, Proc. 24th IEEE Instrumentation and Measurement Technology Conference (IMTC 2007), Warsaw, Poland, 1-3 May 2007, pp. 1-5. DOI: $10.1109 /$ IMTC.2007.379434

[28] R. Morello, C. De Capua, A Measurement System Design Technique for Improving Performances and Reliability of Smart and Fault-Tolerant Biomedical Systems, Lecture Notes in Electrical Engineering, Eds. A. Lay-Ekuakille, S. C. Mukhopadhyay, Vol. 75, 2010, pp. 207-217.

[29] M. Inoue, S. Iwamura, M. Yoshida, EGG measurement under various situations, Proc. 23rd International Conference of the IEEE Engineering in Medicine and Biology Society, Vol.4, 2001, pp. 3356-3358. DOI: $10.1109 /$ IEMBS.2001.1019546

[30] B. O. Familoni, T. L. Abell, K. L. Bowes, A model of gastric electrical activity in health and disease, IEEE Transactions on Biomedical Engineering, Vol. 42, Issue 7, 1995, pp. 647-657. DOI: $10.1109 / 10.391163$

[31] Wei Ding, Shujia Qin, Lei Miao, Ning Xi, Hongyi Li, Yuechao Wang, Processing and analysis of bio-signals from human stomach, Proc. IEEE International Conference on Robotics and Biomimetics (ROBIO), Tianjin, China, December 2010, pp. 769772. DOI: $10.1109 /$ ROBIO.2010.5723423

[32] J. Garcia-Casado, J. L. Martinez-De-Juan, J. L. Ponce, Noninvasive measurement and analysis of intestinal myoelectrical activity using surface electrodes, IEEE Transactions on Biomedical Engineering, Vol. 52, no. 6, 2005, pp. 983-991. DOI: $10.1109 /$ tbme.2005.846730

[33] I. V. Tchervensky, R. J. de Sobral Cintra, E. Neshev, V. S. Dimitrov, D. C. Sadowski, M. P. Mintchev, Centre-specific multichannel electrogastrographic testing utilizing wavelet-based decomposition, Physiological Measurement (IOP SCIENCE), Vol. 27, No. 7, 2006, pp. 569-584. DOI: $10.1088 / 0967-3334 / 27 / 7 / 002$

[34] R. J. Sobral Cintra, I. V. Tchervensky, V. S. Dimitrov, M. R. Mintchev, Optimal wavelets for electrogastrography, Proc. 26th International Conference of the IEEE Engineering in Medicine and Biology Society, San Francisco, CA, 1-5 September 2004, pp. 329-332. DOI: $10.1109 /$ IEMBS.2004.1403159

[35] S. Casciaro, F. Conversano, L. Massoptier, R. Franchini, R. Casciaro, A. Lay-Ekuakille, A Quantitative and Automatic Echographic Method for Real-Time Localization of Endovascular Devices, IEEE Transactions on Ultrasonics, Ferroelectrics, and Frequency Control, Vol. 58, n.10, pp. 2107-17, 2011. DOI: $\underline{10.1109 / T U F F C .2011 .2060}$

[36] S. Urooj, M. Khan, A. Ansari, A. Lay-Ekuakille, A. K. Salhan, Prediction of Quantitative Intrathoracic Fluid Volume to Diagnose Pulmonary Edema Using Labview, Computer Methods in Biomechanics and Biomedical Engineering, 2011, pp.1-6. DOI: $\underline{10.1080 / 10255842.2011 .565054}$ 